\title{
Evaluación de las Competencias Cognitivas y Sociales de Niños que han estado en Situación de Calle
}

\author{
EVALUATION OF COGNITIVE AND SOCIAL COMPETENCES \\ IN C HILDREN THAT HAVE BEEN IN THE STREET
}

\author{
Cuadro, A.; Barg, G.; Navarrete, I.; Suero, M. \\ Universidad Católica del Uruguay, Uruguay
}

\begin{abstract}
Resumen: En el siguiente estudio se busca una aproximación exploratoria a la población de los niños y niñas en situación de calle de Montevideo. Se trabajo con 27 niños de ambos sexos de entre 7 y 13 años. Se consideraron las variables sociodemográficas y se evaluó el desarrollo neuropsicológico, incluyendo la utilización del lenguaje oral, la relación con el código escrito, las habilidades de razonamiento lógico-matemático, el funcionamiento cognitivo general y la exploración de algunos indicadores psicopatológicos. Los resultados muestran que se trata de una población con un alto nivel de carencias y vulnerabilidades, y una importante presencia de trastornos comportamentales. En el aspecto cognitivo, se encuentra algún nivel de efectividad en la ejecución intelectual de operaciones no verbales. En las tareas verbales, se comprobaría un déficit significativo por el escaso desarrollo general del lenguaje. Esto tendría consecuencias negativas en la inserción escolar así como en la capacidad de auto-regulación del comportamiento.
\end{abstract}

Palabras clave: Niños de la calle; Competencia mental; Desarrollo infantil

\begin{abstract}
The following study is an exploratory approach to the population of street children of Montevideo. It involves 27 children of both sexes between 7 and 13 years. Sociodemographic variables were considered and it was evaluated the neuropsychological development, including the use of oral language, the relationship with the writing code, logical-mathematical reasoning skills, cognitive functioning and exploring some psychopathological indicators. The results show that this is a population with high levels of weaknesses and vulnerabilities, and a significant presence of behavioral disorders. In the cognitive aspect, there is some level of effectiveness in the implementation of intellectual nonverbal operations. In the verbal tasks, significant deficits for the low overall development of language were found. This would have a negative impact on school integration as well as on the capacity for self-regulation of behavior.
\end{abstract}

Key words: Homeless children, mental competence, Child development

\section{INTRODUCCIÓN}

La población de niñas y niños en situación de calle ha pasado a ser en los últimos años preocupación de varias instituciones y organizaciones publicas y privadas, ya que cons-tituyen un grupo sometido a una multiplicidad de violencias y necesidades, que ponen en riego su adecuado desarrollo y en tela de juicio el status sanitario social en su conjunto.

Fruto de esta interacción entre actores comunitarios, públicos y académicos se ha comenzado a desarrollar líneas de investigación que den herramientas para conocer mejor la situación. Por ejemplo, el Primer Censo de los Niños de la
Calle (Vara Horna / Asociación por la Defensa de las Minorías, 2001) realizado en Lima, Perú sobre una muestra de 134 niños y niñas en situación de calle, reveló en forma sistematizada algunas características de esta población en relación a lazos familiares, intereses, proceso educativo, internaciones, adicciones, maltrato, asistencia en salud, etc. Dentro de esta línea de investigación latinoamericana se inscribe el presente trabajo. El mismo fue posible gracias a la articulación con el equipo de la ONG El Abrojo que venía trabajando hace varios años con la población objetivo.

Un primer aspecto de impor-tancia tiene que

Correspondencia: Prof. Dr. Ariel Cuadro, Facultad de Psicología, Universidad Católica del Uruguay. Correo Electrónico: acuadro@ucu.edu.uy 
ver con el marco conceptual desde donde esta investigación buscó comprender el funcionamiento psicológico y social de estos niños. Se intentó trazar las trayectorias de desarrollo de este conjunto de niños (Cicchetti, 2006) evitando una aproximación exclusi-vamente basada en el diagnóstico. Mientras que esta última aproximación se conforma con la adjudicación de casos a categorías diagnósticas preestablecidas, la opción elegida entien-de el desarrollo como un camino evolutivo de integración personal de experiencias internas y externas. El sujeto es un agente activo de procesamiento de esa realidad; procesamiento en el cual interactúan factores biológicos, psicológicos y culturales. Cicchetti (2006) caracteriza este enfoque, que denomina psicopatología del desarrollo, como una aproximación basada en el estudio del interjuego de la normalidad y la patología, y por un énfasis en entender los procesos adaptativos y desadaptativos a partir del desarrollo evolutivo.

En este marco, la clasificación diagnóstica no se convierte en un objetivo de investigación en si mismo, sino que se busca construir un perfil de desarrollo de esta población que de cuenta de sus déficit, vulnerabilidades y potencialidades.

La bibliografía da cuenta de numerosas vulnerabilidades en el perfil psicológico de poblaciones similares a la que estudiaremos. Una de ellas tiene que ver con la presencia mayor de psicopatología general. En una investigación que siguió el desarrollo de una muestra de sujetos de diverso nivel socioeconómico desde los 8 a los 40 años en EEUU, se observó una estrecha correlación entre el menor nivel socioeconómico y el aumento de psicopatología, así como una aso-ciación del primero con menores niveles de remisión (Wadsworth y Achenbach, 2005). Estudios de este tipo se han replicado en poblaciones de niños en Latinoamérica. En Brasil se han desarrollado algunas investigaciones especialmente rigurosas sobre este tema. Fleitlich-Bilyk y Goodman (2001), reportan que el nivel de trastornos mentales en las áreas urbanas pobres de Campos de Jordao (San Pablo) se acercaría al $22 \%$, mientras que en las áreas urbanas centrales y rurales llegaría al $12 \%$. Por otra parte, Cucchiaro y Dalgalarrondo (2007) compararon una muestra de niños escolarizados de áreas pobres de Campiñas con una muestra control del centro de la ciudad. Los niños estudiados presentaron un nivel de patología general similar a la muestra control.
Sin embargo, su nivel de trastornos de conducta fue marcadamente superior. La relación del nivel socioeconómico de los niños con la presencia de psicopatología, es esperable si se tiene en cuenta las situaciones evolutivas traumáticas por las que probablemente hayan tran-sitado así como las carencias fami-liares, materiales y culturales que han padecido. Por otra parte, un grado de comportamiento considerado antisocial, puede ser adaptativo en situación de calle.

Dentro del análisis del funcionamiento cognitivo de poblaciones similares a la estudiada, resulta relevante la bibliografía existente sobre el estudio de la función lingüística. Moreno, Rabazo y García-Baamonde (2006) estudiaron las características morfológicas, sintácticas y pragmáticas en el lenguaje oral de niños de 6 a 18 años institucionalizados en Extre-madura, España, a través de la batería BLOC. Además, investigaron el estilo cognitivo a través del MFF-20. De acuerdo a sus resultados, los niños presentaban déficit importantes en el uso del lenguaje debido a una estructura morfológica limitada. Esto generaba una gran dificultad para utilizar el lenguaje como herramienta en diversas situaciones sociales. Por ejemplo, para pedir información, hacer demandas directas e indirectas, expresar acuerdo, mostrar desagrado (especialmente con figuras adultas de autoridad), para utilizar formas verbales irregulares pasadas y futuras y para utilizar pronombres personales, reflexivos y posesivos. Esta utilización del lenguaje limitada correlaciona altamente con un estilo cognitivo general impulsivo, caracterizado por tiempos menores de latencia y mayor número de errores cometidos. Esta estructura de utilización del lenguaje oral tiene consecuencias nefastas para el proceso de escolarización ya que las funciones dañadas son precisamente las que permiten obtener información detallada, describir situaciones y analizar y resolver problemas. Por lo cual, son las habilidades lingüísticas más requeridas por el sistema educativo formal. Además, junto con el aspecto fonológico, habitualmente deficitario en estos contextos (Bravo Valdivieso, 1993), son la plataforma para la adquisición de la lectura y la escritura. De esta forma, dichas carencias generan una brecha entre los recursos cognitivos del sujeto y las demandas del sistema escolar.

Por otra parte, el desarrollo del lenguaje tiene una función clave en la capacidad de auto- 
regulación del comportamiento. De acuerdo a Vygotsky (1999), el lenguaje se convierte en un mecanismo de ensayo y error mental, que evita actuar todas las alternativas. Esto hace posible la evaluación de las consecuencias de los distintos cursos de acción, permitiendo la planificación del comportamiento. Según Luria (1985) este proceso comenzaría a los 3-4 años, estabilizándose como diálogo interno a los 6-7. Ripley y Yuill (2005) chequearon esta hipótesis en un grupo de niños no escolarizados en el Reino Unido. Encontraron una estrecha relación entre las alteraciones en el lenguaje (fundamentalmente expresivo) que tenían estos niños y la ocurrencia de déficit atencional, labilidad emocional, impulsividad y altos niveles de activación. Por otro lado, encontraron un rendimiento superior en la ejecución de tareas no-verbales y un nivel general de psicopatología bastante alto.

En conclusión, la investigación propuseta busca una aproximación exploratoria a las estrategias cognitivas y de interacción social de los niños y niñas que han estado en situación de calle en Montevideo (Uruguay).

\section{OBJETIVOS}

- Describir algunas variables sociodemográficas y del desarrollo de niños que han estado en situación de calle.

- Establecer un perfil de desarrollo que incluya los rasgos principales de sus probables y posibles trayectorias de desarrollo

- Contribuir a que las instituciones u organizaciones brinden los elementos nece-sarios a estos niños y sus familias para un mejoramiento de dichas trayectorias evolutivas.

\section{METODOLOGÍA}

\section{Sujetos}

Participaron 27 niños de ambos sexos (6 niñas y 21 niños) de entre 7 y 13 años.

Los niños que fueron incluidos pertenecían a las siguientes zonas:

- Zona 1: La teja, La Paloma, La Boyada, Cerro Norte (5 niños)

- Zona 2: Colón, Sayago, Lanús (5 niños)

- Zona 3: Lavalleja, Piedras Blancas, Casavalle, Punta Rieles (17 niños).

Estos niños fueron seleccionados a partir de su participación en el Programa Infacalle (Infamilia- Mides- El Abrojo), encontrándose en el momento de la investigación en la segunda fase del mismo. En la primera fase (2005-2006) se realizó la detección de los niños en los lugares donde realizaban sus actividades de calle y se conectó a sus respectivas familias. En la segunda fase (2007) se trabajó con los niños y sus familias con el objetivo de lograr que abandonaran gradualmente la calle. Para ello se apoyó económicamente a las familias, se les facilitó el acceso a la documentación, se promovió la reinserción educativa de los niños, y se los asesoró para que accedieran a otras prestaciones sociales. De manera que cuando se realizó la investigación (segundo semestre del 2007), muchos niños integrantes de la muestra habían abandonado recientemente su actividad de calle, mientras que otros con-tinuaban desarrollándola pero con menor intensidad.

En relación a los criterios de inclusión, la totalidad de los niños de la muestra pertenecieron a los perfiles I y II definidos por el Programa de Infancia, Adolescencia y Familia (Infamilia-Inau, 2007). El perfil de tipo I está conformado por niños que realizan actividades recreativas y/o laborales (mendicidad, clasificación, etc) en la calle de su propio barrio, sin control adulto y exponiéndose a situaciones riesgosas. El perfil de tipo II esta compuesto por niños que realizan actividades de calle fuera de su barrio, en general de tipo laboral y junto a referentes adultos (ej, mendicidad puerta a puerta o trabajo en semáforos). En ocasiones su actividad laboral ha provocado el abandono de la escuela. Finalmente, los niños que integran el perfil de tipo III son niños que viven en la calle, que no tienen vínculos estables con referentes adultos y que muchas veces realizan conductas delictivas como estrategia de supervivencia. Estos niños están en un nivel extremo de vulnerabilidad.

Dado que los niños con los que se trabajó pertenecieron al perfil I y II todos poseían un vínculo más o menos estable con su familia y un cierto nivel de escolarización. Además, este proceso fue reforzado por las intervenciones realizadas por El Abrojo en los dos años anteriores (primera fase del Programa Infacalle)

\section{INSTRUMENTOS}

Protocolo de Derivación: indaga datos demográficos dividida en 9 apartados que corresponden a información general del entrevistado; redes sociales; habilidades cognitivas; habilidades sociales y expectativas futuras; consumo de sustancias psicoactivas; salud general; características de la familia de proce- 
dencia; situación de calle; atención ins-titucional y un último apartado abierto a destacar alguna característica sobresaliente que no hubiera sido mencionada previamente.

Entrevista: Se realizaron entre-vistas abiertas y estructuradas con algunos de los Educadores y con referentes familiares. En algunos casos, se adjuntó el informe de otros técnicos. Los objetivos de estas entrevistas fueron conocer las carac-terísticas generales del desarrollo del niño, los hechos más importantes de la historia familiar y la percepción actual de padres y educadores del mismo.

CUMANIN (Portellano, J.; Mateos, R.; Martinez, A., 2002): El Cuestionario de Madurez Neuropsicológica Infantil tiene por objetivo evaluar el grado de madurez neuropsicológica alcanzada por el niño, así como la posible presencia de signos de disfunción cerebral. Edad de aplicación de 36 a 78 meses (3 y 6 años) con una duración variable de 30 a 50 minutos. Compuesto por 8 escalas principales (Psicomotricidad, Lenguaje Articulado, Lenguaje Comprensivo, Lenguaje Expresivo, Estruc-turación Espacial, Visopercepción, Memoria Icónica y Ritmo) y 5 escalas auxiliares (Atención, Fluidez Verbal, Lectura, Escritura y Late-ralidad).

WISC III (Weschler, 1997): Test de inteligencia para niños de Wechsler de utilización clínica para evaluar la capacidad intelectual de niños de 6 años a 16 años y 11 meses de edad. Consta de varias subpruebas, relacionadas con una habilidad o capacidad básica y que forman parte de una escala más extensa (capacidad cognitiva general). En este estudio se aplicaron las subpruebas Analogías, Aritmética y Dígitos, con el fin de evaluar en los sujetos la capacidad de análisis y abstracción; de concentración, memoria y de conocimiento matemáticos básicos; y de memoria (auditiva) a corto plazo.

SDQ (Goodman, 1997; 2001): Cuestionario de Fortalezas y Dificultades. Puede ser aplicado a los padres, tutores o profesores de niños de 4 a 16 años y a los jóvenes de entre 11 y 16 años. La escala para jóvenes está compuesta por los factores: estrés general; angustia emocional; dificultades de conducta; hiperactividad y dificultades de atención; atención y actividad; dificultades para llevarse bien con otros jóvenes; comportamiento amable y servicial; impacto de dificultades en la vida del joven.

Se consideró especialmente la utilización de este instrumento porque brinda una visión global del patrón de relacionamiento social de cada niño. Las subescalas de comportamiento prosocial, problemas con pares y dificultades de conducta evalúan específicamente esta dimensión. Por otra parte, el resto de las sub-escalas ofrecen índices básicos acerca de problemas emocionales que, de estar presentes, afectan los vínculos de manera significativa.

\section{PROCEDIMIENTOS}

Los niños y niñas concurrían semanalmente a sus respectivas sesiones de evaluación, acompañados por familiares o por los educadores responsables.

Para todas las evaluaciones se planteó inicialmente la aplicación de todas las técnicas previstas, sin embargo, las mismas fueron adaptadas contemplando algunas necesidades individuales de los jóvenes.

Los grupos evaluadores estaban conformados por uno o dos técnicos, quienes se encargaban del trabajo directo con los niños, más un equipo de 3 coordinadores quienes su-pervisaban su labor.

\section{RESULTADOS}

La muestra estaba conformada por niños de ambos sexos (22.2\% niñas $77.8 \%$ niños), con edades comprendidas entre los 7 y 13 años. La mayor prevalencia se ubicó en las edades de 8 y 9 años, con el $26 \%$ y $22 \%$ de los casos respectivamente.

En relación a las variables sociodemográficas, se tomaron aquellas que permiten construir un perfil de los procesos educativos de los niños y sus familias. En cuanto al nivel educativo del referente familiar (Figura 1), encontramos que la mayoría no ha culminado la primaria $(56.4 \%)$, y en muy pocos casos se han cursado niveles superiores de enseñanza (12.9\% ha cursado, por lo menos algún año de secundaria). El nivel de analfabetismo de los referentes adultos de estos niños es ampliamente superior al nivel de analfabetismo de la población general.

Los niños han cursado uno o tres años de enseñanza formal (Figura 2), sin embargo la mayoría ha alcanzado el nivel escolar de primer o segundo año (Figura 3). Es de destacar cómo algunos jóvenes han cursado seis, siete y hasta ocho años de enseñanza formal y sin embargo, ninguno ha superado quinto año de primaria. La mayor concentración de niños tiene dos años de rezago (Figura 4). 


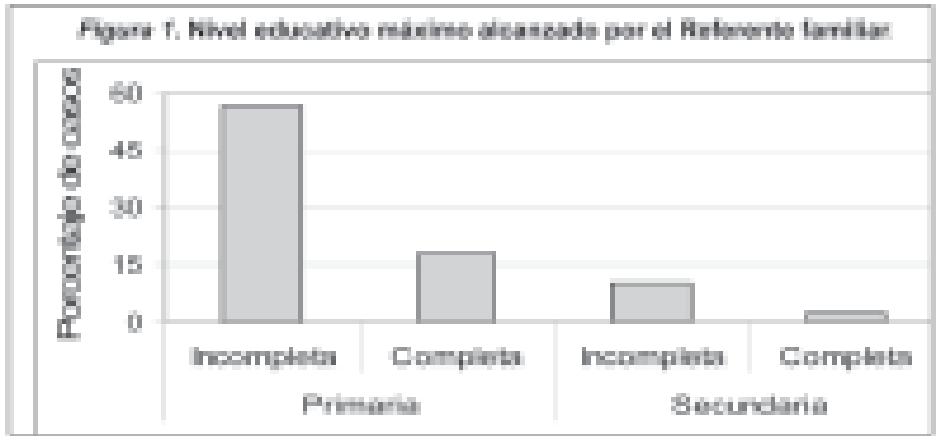

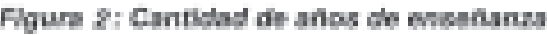

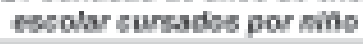

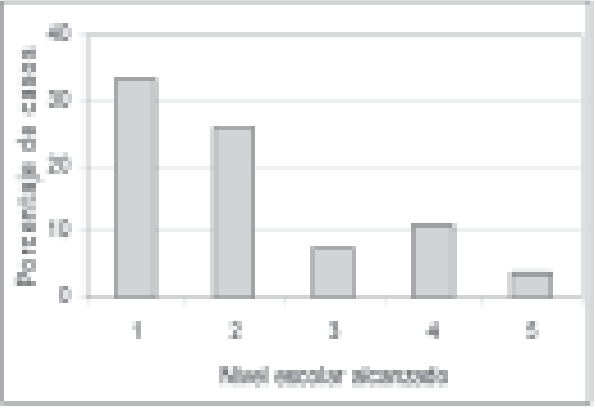

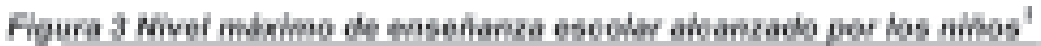

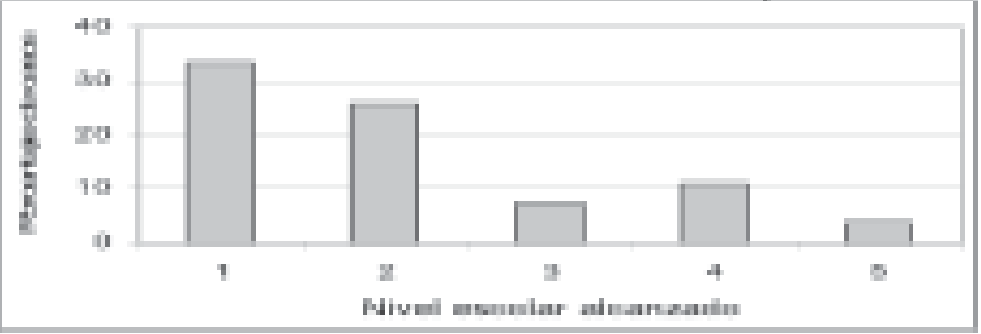

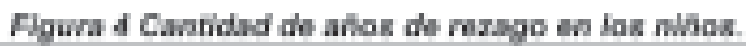

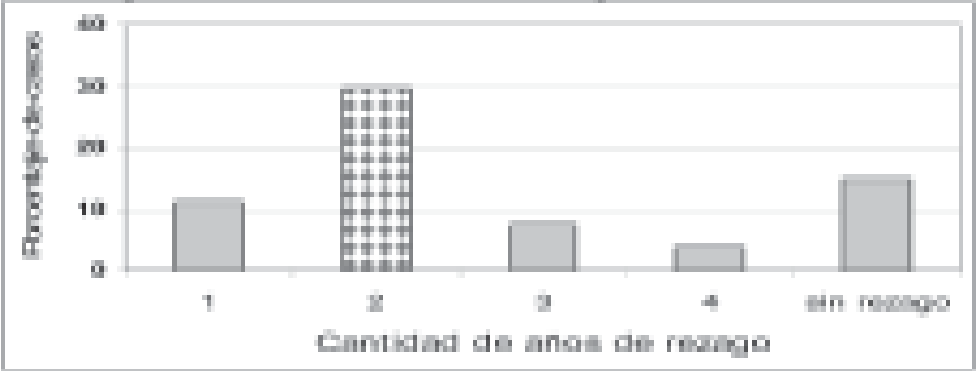

Por otra parte, un alto porcentaje de la población realiza actividades de calle. Tal como puede verse en la Figura 5 la mayoría de los jóvenes (91.8\%) practica, por lo menos, un tipo de actividad en la calle, destacándose la

El nivel escolar hace referencia a los años de la enseñanza primaria, 1 = Primero; 2 = Segundo; 3 = Tercero; 4 = Cuarto; $5=$ Quinto

${ }^{2}$ REC = Recolección de residuos; MEND = Mendicidad; RECREA = Recreativa; $C O M=$ Comunitaria; TRAB = Trabajo infantil; mendicidad (46.9\%) dentro de éstas.

En relación a las variables psicológicas, se evaluó el perfil de desarrollo neuropsicológico general. En la prueba CUMANIN (Portellano, Mateos y Martínez, 2002) (Figura 6), las escalas con valores más elevados fueron Estructuración primaria, 1 = Primero; 2 = Segundo; 3 = Tercero; 4 = Cuarto; 

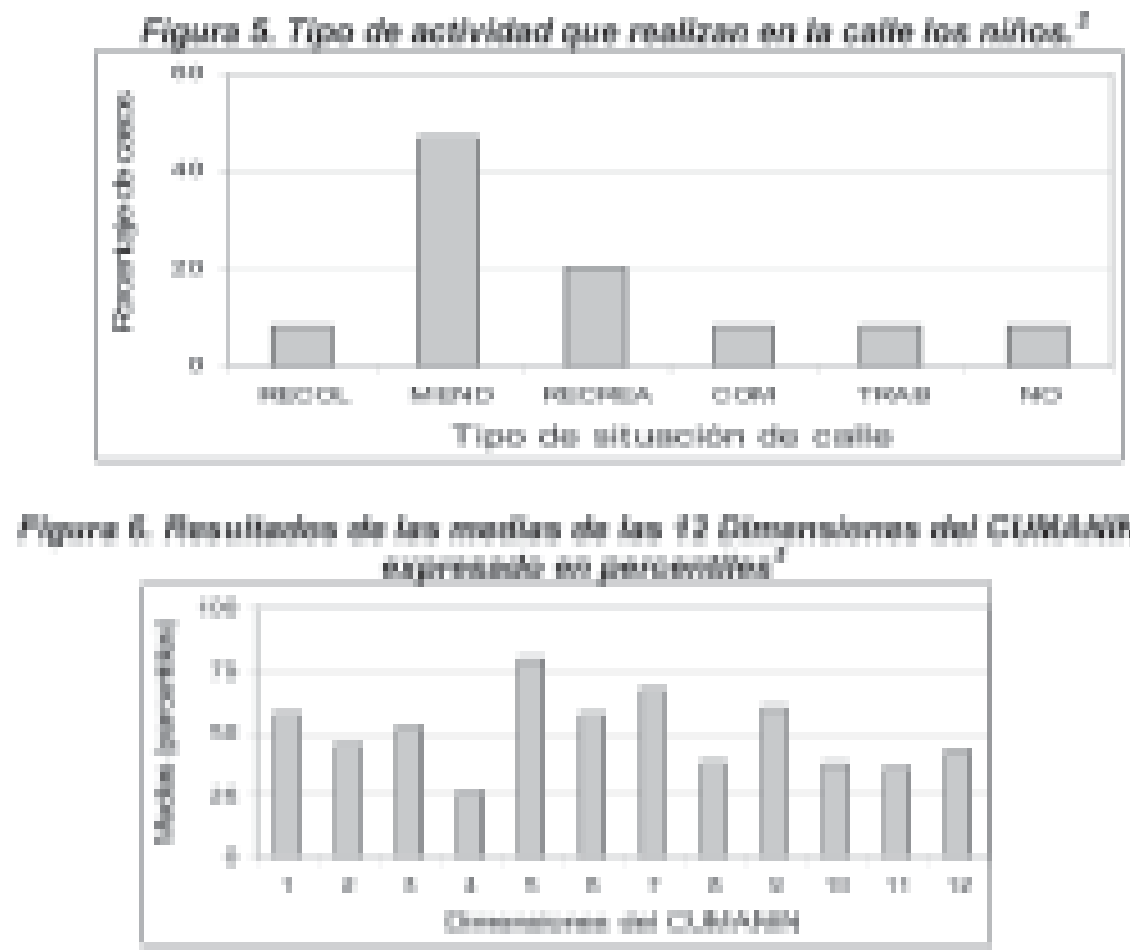

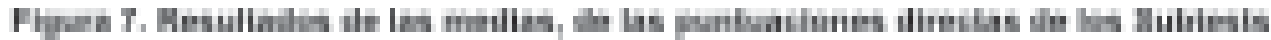

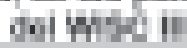

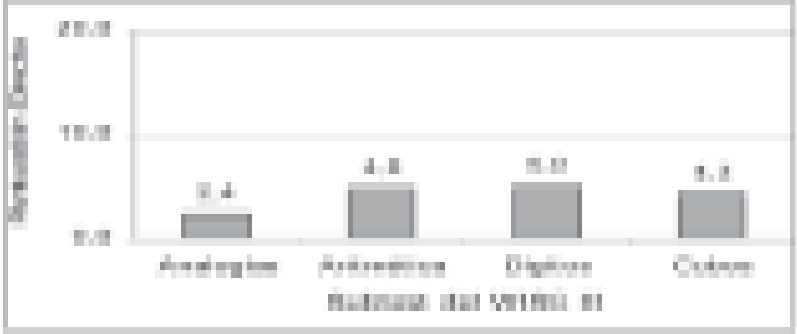

espacial, Memoria icónica, Fluidez verbal, Visopercepción y Psicomotricidad. Por otro lado, las escalas con valores más descendidos fueron Lenguaje com-prensivo, Lectura, Atención, Ritmo.

Se administraron algunos componentes del WISC-III (Weschler, 1997) para evaluar los aspectos vinculados a la capacidad cognitiva general, a procesos básicos y conocimientos matemáticos. En los subtest del WISC, tal como se muestra en la Figura 7, se obtuvieron valores significativamente por debajo de lo esperado para sus respectivas eda-des, inclusive por debajo del margen de la desviación estándar.

Los resultados del Cuestionario de Capacidades y Dificultades SDQ (Goodman, 1997, 2001) se presentan en la Tabla 1.

\section{DISCUSIÓN Y CONCLUSIONES}

El estudio realizado buscó una aproximación exploratoria a la población de los niños y niñas en situación de calle de Montevideo con los cuales trabaja la ONG El Abrojo. Los niños en

$\mathrm{NO}=$ No realiza trabajo alguno

31 = Psicomotricidad; 2 = Lenguaje articulatorio; 3 = Lenguaje expresivo; 4 = Lenguaje comprensivo; 5 Estructuración espacial; 6 = Visopercepción; 7 = Memoria icónica; 8 = Ritmo; 9 = Fluidez verbal; $10=$ Atención; 11 = Lectura; $12=$ Escritura. 


\begin{tabular}{|c|c|c|c|}
\hline & Martal & Inh & Áminal \\
\hline hrptodn & Hh & IAh & $4 h$ \\
\hline $\begin{array}{l}\text { BHrulads dy } \\
\text { comductur }\end{array}$ & 74 & nit & Wh \\
\hline Hiphatshis & 41. & 4 & $4 x$ \\
\hline $\begin{array}{l}\text { Probann } \\
\text { butpuses }\end{array}$ & Mh & Hh & nh \\
\hline Prominil & 417 & 14h & 144 \\
\hline Tolit dituhut & IA & Mh & HW \\
\hline
\end{tabular}

situación de calle son una población escasamente estudiada a nivel internacional, regional y nacional. Esto se debe a la dificultad para tomar contacto con los niños y establecer un vínculo de trabajo consensuado y motivador. En esta ocasión, el trabajo previo de los equipos técnicos en campo de El Abrojo proporcionó un sostén que hizo posible la realización de los estudios diagnósticos. Por estas razones, el principal objetivo de esta investigación consistió en describir algunas variables e indicadores de desarrollo en la población estudiada. Estas variables fueron fundamentalmente psicológicas, aunque se incluyeron algunas de corte sociodemográfico. Entre las primeras, cuya indagación constituye el aporte más significativo de este estudio, se incluyen el patrón de desarrollo neuropsicológico, la utilización del lenguaje oral, la relación con el código escrito, las habilidades de razonamiento lógico-matemático, el funcionamiento cognitivo general y la exploración (screening) de algunos indicadores comportamentales. El relevamiento de las variables sociodemográficas así como las entrevistas con niños/as, familiares y educadores permitieron una mayor comprensión del contexto en el que crece cada niño. A partir de la indagación de estas variables se buscó establecer relaciones de conjunto que aporten a la configuración de un perfil de desarrollo de estos niños, que incluya los rasgos principales de sus probables y posibles trayectorias de desarrollo (Cichetti, 2006) individuales y colectivas. Con la esperanza de que estos resultados contribuyan a que las instituciones educativas y promocionales (públicas y comunitarias) brinden los elementos necesarios a estos niños y sus familias para incluir la dimensión de lo deseable en dichas trayectorias evolutivas.

Antes de ingresar a la discusión de los resultados obtenidos, resulta pertinente detenerse en algunas consideraciones de orden metodológico. En primer lugar, lo limitado del tamaño muestral de la población estudiada $(n=27)$ y la heterogeneidad de alguna de sus características (por ejemplo, la edad). Además, la selección de la misma se realizó obedeciendo a criterios de interés para la intervención social que El Abrojo realiza con estos niños, por lo que no se utilizaron procedimientos que habiliten la generalización de los resultados en relación a poblaciones mayores (tamaño, aleatorización, representatividad, control). De esta forma, se priorizó la participación de los niños que requerían una evaluación para mejorar su procesos de inserción escolar y social. En segundo lugar, se utilizaron técnicas de evaluación que en su mayoría no están diseñadas específicamente para la población estudiada. Si bien estos elementos limitan la generalización estadística de los datos, consideramos que el mayor aporte de esta investigación consiste en explorar los aspectos estudiados en una población de difícil acceso y, a partir de la descripción obtenida, generar hipótesis de investigación a ser confrontadas en estudios cuantitativamente mayores.

Como se puede observar en la sección Resultados se trabajó con niños y niñas cuyas edades van desde los 7 a los 13 años, concentrándose la mayor cantidad en los 8 y 9 años y distribuyéndose luego en el resto de las edades. Cuando se relaciona esta distribución etaria con los trayectos realizados en el sistema educativo se constata un desfasaje considerable. El $60 \%$ de los niños se agrupa en el primer y segundo 
grado, existiendo rezago escolar en la mayoría de los casos (la mayoría de los niños repitió 2 o más años). Cuando se evalúa la adquisición de las habilidades de lectura y escritura se ve que no hay una relación clara entre la cantidad de años cursados en la escuela, e incluso el grado escolar alcanzado, y el mejor dominio de la lectura y la escritura. El nivel lector de estos niños es prealfabético y lo mismo sucede con la escritura ${ }^{4}$. Es decir, que para esta población de niños y niñas en situación de calle el hecho de ir a la escuela, cursar y pasar de grado no implica necesariamente un avance en su proceso de alfabetización. Otro elemento significativo que surge del análisis de los datos socio-demográficos tiene que ver con el nivel educativo alcanzado por lo padres. El 56,4\% de los referentes adultos (padre, madre, pareja de los mismos, abuelos, etc) de los niños no han finalizado la enseñanza primaria. El nivel de analfabetismo de los padres supera ampliamente al de la población general y da cuenta de un circuito de reproducción de la exclusión del sistema educativo sumamente consolidado. Las investigaciones sobre el tema dan cuenta de la importancia de la estimulación en el hogar en la adquisición y afianzamiento del lenguaje oral y escrito (Bravo Valdivieso, 1993, 1995).

A nivel de la evaluación del desarrollo madurativo neuropsicológico general, se observan mejores resultado en las áreas vinculadas a la ejecución de actividades (Índice Ejecutividad). Particularmente las áreas de psicomotricidad, viso-percepción ${ }^{5}$, memoria visual y estructuración espacial que darían cuenta de que algunas capacidades cognitivas básicas no están dañadas estructuralmente. Estas habilidades son condiciones necesarias para avanzar en un proceso de escolarización y su ausencia o daño profundo le impondría severas restricciones al mismo.

La capacidad de manejar el lenguaje oral es una de las áreas en que estos niños/as presentan descenso significativo; el nivel alcanzado les permite mantener una conversación, pudiendo entender e interactuar con cierto grado de fluidez. No obstante lo cual, se observa un vocabulario extraordinariamente limitado así como estructuras morfológicas sumamente pobres, lo cual concuerda con estudios realizados en otros países con poblaciones similares (Moreno, Rabazo y García, 2006). En relación al razonamiento lógico-matemático y las habilidades invol-ucradas en el mismo, el desarrollo observado es claramente inferior al esperable de acuerdo a la edad, a la cantidad de años cursados y al grado escolar alcanzado. Los indicadores más positivos, aunque también bajos, se refieren al manejo matemático elemental (Aritmética del Wisc-III). Es posible además que las actividades realizadas en la calle, fundamentalmente las que implican manejo del dinero (venta en ómnibus, mendicidad, clasificación) proporcionan a estos niños el concepto de número así como algunos mecanismos de cálculo intuitivos; así como podrían explicar la capacidad de manejo verbal, como comprender e interactuar en una conversación en función de un objetivo concreto. Sin embargo, en ninguno de los dos aspectos señalados (razonamiento lógico-matemático y lenguaje oral) existe un desarrollo formal acorde a su edad, probablemente vinculado a la ausencia de estimulación en el hogar y al escaso proceso de alfabetización de estos niños en las escuelas.

Los resultados de la evaluación de la capacidad atencional también están marcadamente descendidos (percentil 37, Cumanin). La capacidad atencional es una habilidad clave en la adquisición de conocimientos y es uno de los recursos cognitivos que son más requeridos por el sistema escolar.

Las grandes deficiencias en la adquisición y manejo del lenguaje (oral y escrito) que se observan en estos niños y niñas, de seguro inciden fuertemente en su capacidad de aprendizaje y en su adaptación al sistema educativo formal. El lenguaje permite que el niño pueda interactuar adecuadamente en un ambiente que se maneja básicamente a través del código escrito, tanto desde el punto de vista social (normas, comportamiento en clase, horarios, etc) como académico. También condiciona la capacidad de aprendizaje general ya que el manejo del lenguaje tiene un efecto "en cascada" sobre todos los aprendizaje, la palabra oral y escrita es el vehículo privilegiado de construcción de conocimiento. Finalmente, el manejo del lenguaje es un elemento clave en la autoregulación del comportamiento. El lenguaje permite una sucesión

\footnotetext{
${ }^{4}$ Tal es así, que algunas pruebas que investigan la lectura para escolares TECLE (Marin y Carrillo, 1997), LEE (Delfior y col. 2005) que fueron incluidas en la planificación de la batería de evaluación, en la práctica no pudieron ser administradas ya que no existía el nivel mínimo requerido para hacerlo.

${ }^{5}$ Capacidad de coordinación entre la percepción visual y la ejecución de una tarea motriz fina.
} 
de diálogos internos a través de los cuales la persona va desplegando en su mente los cursos de acción posibles, analizando rápidamente las consecuencias negativas y positivas, y eligiendo la opción ponderada como más adecuada. Este proceso, que puede durar microsegundos y que está fuertemente influido por elementos emocionales, es fundamental para la auto-regulación del comportamiento. Permite la elección de estrategias más reflexivas y la inhibición de comportamientos impulsivos (Moreno, Rabazo y García, 2006). El deterioro de esta capacidad en la población estudiada genera consecuencias negativas en lo que se refiere a adaptación a normas y al relacionamiento interpersonal, especialmente con pares.

Dentro de la exploración realizada a través de la escala SDQ, la subescala referida a Problemas de Conducta fue una de las que presentó un puntaje más elevado. Por lo expresado anteriormente, se puede hipotetizar una relación entre deficiencias en el manejo del lenguaje y problemas de adaptación a normas y relacionamiento interpersonal. Esta relación ha sido constatada en otras investigaciones con poblaciones similares (Ripley y Yuill, 2005). Obviamente, en esta área se deben considerar los aspectos ambientales y evolutivos. En relación a los primeros, es importante recordar que la situación de calle expone a estos niños a una enorme variedad de situaciones peligrosas y violentas. En este marco, algunas conductas disruptivas en relación a las normas y la agresividad podrían formar parte de estrategias cotidianas de supervivencia. Por otra parte no deben dejar de valorarse la secuencia de sucesos estresantes y traumáticos que han vivido los niños y niñas objeto de la evaluación. Estos sucesos traumáticos incluyen desde violencia, maltrato, abuso, insatisfacción de las necesidades básicas, abandono de los padres, desempleo de los mismos, agresiones de pares y adultos, etc. Por otra parte, se constatan niveles elevados de Hiperactividad y Angustia. Los valores elevados en esta última sub-escala son coherentes con la historia de sucesos traumáticos citada anteriormente. Asimismo, es muy importante el déficit en comportamiento Prosocial, elemento que puede dificultar en gran medida la inserción educativa de estos niños. Con lo cual ésta se vería obstaculizada por cuestiones de relacionamiento además de las dificultades en el proceso de aprendizaje

Los resultados de la exploración realizada, así como las entrevistas clínicas con niños y sus familias, dan cuenta de un nivel de patología general superior al de poblaciones similares de otros países, por ejemplo, Brasil (Cucchiaro y Dalgalarrondo, 2007). Aunque esta investigación no pretendía diagnosticar patologías clínicas específicas, se puede suponer la extrema vulnerabildad de esta población en relación a padecer trastornos por estrés post-traumático, depresión y otros síndromes clínicos; tanto en lo que se refiere a los niños como a sus familias. Esta vulnerabilidad extrema se ve reflejada en la subescala de Dificultades del SDQ, que señala un nivel claramente anormal de las mismas. Obviamente, estas hipótesis deberán ser confirmadas por investigaciones cuyo objeto específico sea el diagnóstico epidemiológico. En este sentido, cualquier intervención que busque impactar en una mejora en la inserción educativa y social de estos niños, debería necesariamente tomar en cuenta algún tipo de abordaje de esta problemática, tanto en relación a los niños como a sus familias. Se ha estudiado que los trastornos psicológicos disparados por estas situaciones traumáticas tienen un efecto negativo sobre las capacidades de aprendizaje en general, y sobre la adquisición y desarrollo del lenguaje en particular (Ripley y Yuill, 2005).

En conclusión, a partir de los resultados analizados se pueden visualizar algunos elementos característicos del perfil de desarrollo de estos niños. En primer lugar, un nivel básico alcanzado de desarrollo neuropsicolgico que le ha permitido el desarrollo de habilidades como el cálculo elemental y la capacidad de comunicarse oralmente. Además de poder suponer que con intervenciones oportunas y que refuercen la estimulación ambiental pueden generarse resultados que favorezcan el desa-rrollo de estos recursos personales.

Por otro lado, se constatan problemas graves en las habilidades cognitivas vinculadas al lenguaje tanto en el aspecto oral (vocabulario, morfología, sintaxis extremadamente pobres) como escrito (lectura y escritura).

Finalmente, un nivel de problemas psicológicos y emocionales elevado, particularmente problemas conductuales, estrechamente relacionado a las carencias ambientales y sucesos traumáticos evolutivos.

\section{REFERENCIAS}

Bravo Valdivieso, L. (1993a). Retardo mental so- 
ciocultural o diferencias cognitivas en niños de nivel socioeconomico bajo. Psicología, 11 (1), 46-66.

Bravo Valdivieso, L. (1995b). Lenguaje y dislexias. Santiago de Chile: Universidad Católica de Chile.

Cicchetti, D. y Cohen, D. J. (2006). Developmental Psychopathology Vol 1. Theory and Method. New Jersey: Jhon Wiley \& Sons.

Cucchiaro, G. y Dalgalarrondo, P. (2007). Saúde mental e qualidade de vida em adolescentes: um estudo entre escolares em dúas áreas urbanas contrastantes. Revista Brasilera de Psiquiatría, 29 (3) :213-221.

Delfior, S.; Fonseca, L.; Gottheil, B.; Aldrey, A.; Pujals, M.; Rosa, G.; Jiménez G.; Serrano, F.(2005) Test LEE. Buenos Aires: Paidos

Fleitlich-Bilyk, B. y Goodman R. (2001) Social factors associated with child mental health problems in Brazil: Cross Sectional Survey, British Medical Journal, 323 (7313):599600.

Goodman, R. (1997a) The strengths and difficulties questionnaire. A research note. J. Child Psychol Psychiat, 38, 581-586

Goodman, R. (2001b) Psychometric properties of the Strengths and Difficulties Questionnaire (SDQ). Journal of the American Academy of Child and Adolescent Psy-chiatry, 40, 1337-1345.

Infamilia-Inau (2007). Dimensionamiento de la situación de calle de niños, niñas y adolescentes. Informe final. Página web de Infamilia. Consultada 15.7.2008 en: http:// www. infamilia.gub.uy/documentoportada.

Luria, A. R. (1985). Lenguaje y pensamiento. Barcelona: Martínez Roca.

Marin y Carrillo (1997) Test de Eficacia Lectora (TEL). Universidad de Murcia

Moreno Manso,J. M., Rabazo Méndez, M.J. y García-Baamonde, M. E. (2006). Competencia lingüística y estilo cognitivo en niños institucionalizados. Revista de Logopedia,
Foniatría y Audiología, 26, 2, 115-125.

Portellano, J.; Mateos, R.; Martinez, A. (2002). CUMANIN (c): Cuestionario de madurez neuro-psicológica infantil. Madrid: TEA.

Ripley, K. y Yuill, N. (2005) Patterns of language impairment and behavior in boys excluded from school. British Journal of Educational Psychology, 75, 37-50.

Vara Horna, A. Asociación por la Defensa de las Minorías (2001). Informe estadístico del Primer Cen-so de Niños de la Calle. Lima. En: http://www.aristidesvara.com/investigaciones/psicología/informe_estadistico/ index.

Vygotsky, L. S. (1999). Pensamiento y lenguaje. Teoría del desarrollo cultural de las funciones psíquicas. Buenos Aires: Fausto.

Wadsworth, E. y Achenbach, T. (2005). Explaining the Link Between Low Socioeconomic Status and Psychopathology: Testing Two Mechanisms of the Social Causation Hypothesis. Journal of Consulting and Clinical Psychology, 73,1146-1153.

Wechsler, D. (1997). Wisc-III. Test de inteligencia para niños. Buenos Aires: Paidós. 